University of San Diego

Digital USD

Spring 5-27-2017

\title{
Implementation of a Metabolic Management Practice Change in a Community Behavioral Health Clinic: An Evidence Based Practice Project
}

Brenda J. Boyle

University of San Diego, brendajboyle@sandiego.edu

Follow this and additional works at: https://digital.sandiego.edu/dnp

Part of the Nursing Commons, and the Psychiatry and Psychology Commons

\section{Digital USD Citation}

Boyle, Brenda J., "Implementation of a Metabolic Management Practice Change in a Community Behavioral Health Clinic: An Evidence Based Practice Project" (2017). Doctor of Nursing Practice Final Manuscripts. 56.

https://digital.sandiego.edu/dnp/56

This Doctor of Nursing Practice Final Manuscript is brought to you for free and open access by the Theses and Dissertations at Digital USD. It has been accepted for inclusion in Doctor of Nursing Practice Final Manuscripts by an authorized administrator of Digital USD. For more information, please contact digital@sandiego.edu. 
Final Manuscript

Implementation of a Metabolic Management Practice Change in a Community Behavioral Health Clinic:

An Evidence Based Practice Project

Brenda Boyle, MSN, PMHNP-BC

brendajboyle@ sandiego.edu 


\begin{abstract}
Purpose: To increase psychiatric providers' adherence to the recommendations set forth in the American Psychiatric Association Consensus Guideline for metabolic management of patients prescribed second generation antipsychotic medications (SGAs).

Background: Research suggests that SGAs increase the indicators of metabolic syndrome and a need exists in collecting patients' biometrics that influences metabolic management.
\end{abstract}

Methods: Guided by the Iowa Model of Research in Practice, baseline data was collected on blood pressure and body mass index documentation. Metabolic management education was provided to providers in a community behavioral health clinic and four weeks of post data was collected.

Results: A 70\% increase in the compliance of blood pressure documentation and a $58.23 \%$ increase in body mass index documentation were noted from the baseline data to the post education data.

Conclusions: This evidenced-based practice project is the first step in the process of shifting psychiatric provider behavior in adhering to the guidelines for metabolic management of the patients prescribed SGAs. 


\section{Background}

Antipsychotic medications are an integral component of treatment for many psychiatric disorders and psychiatric symptoms. Given that treatment for many common psychiatric disorders often requires lifelong psychotropic management and that as a profession, psychiatry often prescribes antipsychotics for off label uses; the demand for antipsychotic medication has significantly increased over the past generation. ${ }^{1,3,4,6}$

Typically prescribed for psychosis, first generation antipsychotics (FGAs) were developed in the 1950's and often referred to as "neuroleptics" because of their capacity to affect psychomotor slowing, emotional quieting and affective indifference. Regrettably, FGAs also have a higher risk of neurological side effects including tardive dyskinesia and extrapyramidal dystonia. ${ }^{1}$

Second generation antipsychotics (SGAs) came onto the market in the 1980's. Also called "atypical", these newer SGAs demonstrated a lower risk of neurological side effects, but in time, were linked with a proven risk of developing metabolic side effects including weight gain with an increase in waist circumference, hyperglycemia,

dyslipidemia subsequently leading to cardiovascular disease and/or diabetes. ${ }^{1,3,4,6}$

Recognizing the severe chronicity of the consequences of the metabolic side effects of the atypical antipsychotic medications, in 2003 the American Diabetes Association, the American Psychiatric Association, the American Association of Clinical Endocrinologists, and the North American Association for the Study of Obesity (ADA/APA/ACE/NAASO) convened at a conference creating a consensus position statement and monitoring screening protocol for patients prescribed SGAs. ${ }^{1}$ 


\section{Purpose}

Staffed with psychiatric physician residents, an attending physician, and a newly hired psychiatric nurse practitioner (PMHNP), the psychiatric behavioral health clinic is currently operational two afternoons a week. Averaging 33 psychiatric visits each afternoon, the current patient volume does not provide justification for the hiring of a medical assistant. Any biometrics or baseline data such as blood pressure, height, weight, or waist circumference needs to be obtained and documented by the clinic psychiatric providers themselves at the time of the psychiatric visit.

The aim of this evidence based practice $(\mathrm{EBP})$ project was to provide improved patient care by increasing the psychiatric providers' adherence to the recommendations created by the Consensus Development Conference on Antipsychotic Drugs, Obesity, and Diabetes for metabolic screening and treatment of patients prescribed SGAs. Psychiatric providers prescribing SGAs should ensure, according to the consensus guidelines established by the conference, that a baseline blood pressure, height and weight for a body mass calculation, and waist circumference measurement are documented in the electronic health record (EHR). ${ }^{1,3,4,11,12}$ 


\section{Synthesis of the Evidence}

A literature search revealed Meta-analysis evidence, as well as individual experimental case and cohort studies to validate the monitoring protocol noted in Table 1; established in 2003 by the Consensus Development Conference on Antipsychotic Drugs and Obesity. $3,4,8,11$

Table $1 \quad$ Metabolic Monitoring Protocol

\begin{tabular}{|l|l|l|l|l|l|l|l|}
\hline & Baseline & 4 weeks & 8 weeks & 12 weeks & Quarterly & Annually & $\begin{array}{c}\text { Q 5 } \\
\text { Years }\end{array}$ \\
\hline $\begin{array}{l}\text { Family } \\
\text { History }\end{array}$ & $\mathrm{X}$ & & & & & $\mathrm{X}$ & \\
\hline $\begin{array}{l}\text { Weight } \\
\text { (BMI) }\end{array}$ & $\mathrm{X}$ & $\mathrm{X}$ & $\mathrm{X}$ & $\mathrm{X}$ & $\mathrm{X}$ & & \\
\hline $\begin{array}{l}\text { Waist } \\
\text { Circumference }\end{array}$ & $\mathrm{X}$ & $\mathrm{X}$ & & & & $\mathrm{X}$ & \\
\hline $\begin{array}{l}\text { Blood } \\
\text { Pressure }\end{array}$ & $\mathrm{X}$ & & & $\mathrm{X}$ & & $\mathrm{X}$ & \\
\hline $\begin{array}{l}\text { Fasting } \\
\text { Plasma } \\
\text { Glucose }\end{array}$ & $\mathrm{X}$ & & & $\mathrm{X}$ & & $\mathrm{X}$ & \\
\hline $\begin{array}{l}\text { Fasting lipid } \\
\text { profile }\end{array}$ & $\mathrm{X}$ & & & $\mathrm{X}$ & & & $\mathrm{X}$ \\
\hline
\end{tabular}

The Conference observed that although trends have been identified linking SGAs and obesity or diabetes, the majority of studies and subsequent data focused on those patients with a diagnosis of schizophrenia. Within the population of patients who carry a diagnosis of schizophrenia, weight gain and accompanying changes in body composition during the psycho-pharmaceutical treatment with SGAs weight gain is significantly evident. The weight gain and physical changes are frequently combined with dyslipidemia and the subsequent development of diabetes. $1,2,3,9,10$

During the course of treatment with SGAs, clinicians who prescribe the SGAs should be monitoring weight through the entire term of treatment. Additionally, the 
baseline and periodic monitoring of blood pressure, waist circumference, and laboratory values should be routinely assessed. . $^{1,8}$

The reasons why SGAs cause metabolic changes is not fully understood. The six SGAs, noted in Table 2, differ in chemical makeup and efficacy and some contribute to metabolic changes more than others. The SGA medications are not created equal nor are they prescribed interchangeably for psychiatric conditions and symptomology. The risk versus benefit to the patient who is prescribed a particular SGA and the "return on investment" in alleviating symptoms needs to be taken into consideration even while monitoring for metabolic shifts. For some, the efficacy of management of the psychiatric symptoms, regardless of the metabolic risks or changes, outweighs any consideration to switch to an alternate medication. ${ }^{4,5,6,8}$

Table 2 Second Generation Antipsychotics

\begin{tabular}{|c|c|c|}
\hline Generic Name & Trade Name & Year Approved \\
\hline Clozapine & Clozaril & 1989 \\
\hline Risperidone & Risperdal & 1993 \\
\hline Olanzapine & Zyprexa & 1996 \\
\hline Quetiapine & Seroquel & 1997 \\
\hline Ziprasidone & Geodon & 2001 \\
\hline Aripiprazole & Abilify & 2001 \\
\hline
\end{tabular}

\section{Practice Change}

The psychiatrist residents as providers at the psychiatric behavioral health clinic were observed to rarely be obtaining a baseline blood pressure on adult patients. The clinic had made available stethoscopes and two manual blood pressure cuffs occasionally used by the psychiatric providers for obtaining a blood pressure reading. The newly adopted EHR did have provision in the psychiatric visit documentation template for the baseline biometrics, yet, because the clinic did not have a scale during the retrospective 
data period, height, weight, or body mass index were not being documented at the time of the psychiatric visit.

Despite the acceptance and acknowledgement of the importance of the guideline, psychiatric clinics as a whole do not appear to be routinely following the ADA/APA/ACE/NAASO guideline. ${ }^{6,11}$ Psychiatrists are often reluctant to collect and document blood pressure, height and weight, and are particularly averse to measuring waist circumference. Provider reluctance appears to be a significant impediment to the overall broad implementation of this clinical guideline. ${ }^{4,6,11}$

\section{Implementation}

The practice change occurred on February 21 and $27^{\text {th }}, 2017$. Education on the ADA/APA/ACE/NAASO guideline to the psychiatric providers occurred on the same day. The post-implementation data was collected from the EHR from February 21, 2017 through March 21, 2017. For comparison, twenty four months of retrospective data from the EHR from July 2014 through July 2016 was collected evaluating patient charts of those prescribed SGAs noting whether blood pressure, BMI, or waist circumference were documented at the psychiatric visit. A letter of support from the clinical director was obtained and the request to initiate a special project was sent through the appropriate channels at the clinic site. The project was submitted through the USD institutional review board (IRB) and approval was obtained under exempt status.

The affected staff included eight psychiatric physician resident providers, two attending psychiatrists, and one PMHNP. The support of the clinic director and of the attending psychiatrists was vital to the success of the project. The Iowa Model of Research in Practice was used as the implementation guide. ${ }^{7}$ 


\section{Outcomes}

Utilizing data from the EHR, the project results were intended to be evaluated on three objectives: a $20 \%$ increase in the documentation rate of blood pressure, a $20 \%$ increase over baseline in the documentation rate of body mass index, and a $20 \%$ increase over baseline in the documentation of waist circumference.

The control chart in Table 3 below compares pre-intervention data with the results of the post intervention data in the blood pressure monitoring rate. The blue dots reveal that $5.31 \%$ of the time during the retrospective data collection period a blood pressure was taken and documented at the time of the psychiatric visit. In other words, a blood pressure was occasionally taken and documented during this time. The red dots falling below the mean flag an undesirable trend noting essentially $0 \%$ of the time was blood pressure was taken and documented at the time of the psychiatric visit. Following the educational intervention to the providers, an upward trajectory is noted during the month of post intervention data collection. The $70 \%$ documentation rate of blood pressure is well above the target outcome of a $20 \%$ increase over baseline.

Table 3

Blood Pressure Monitoring Rate for Patients Receiving Second Generation Antipsychotics

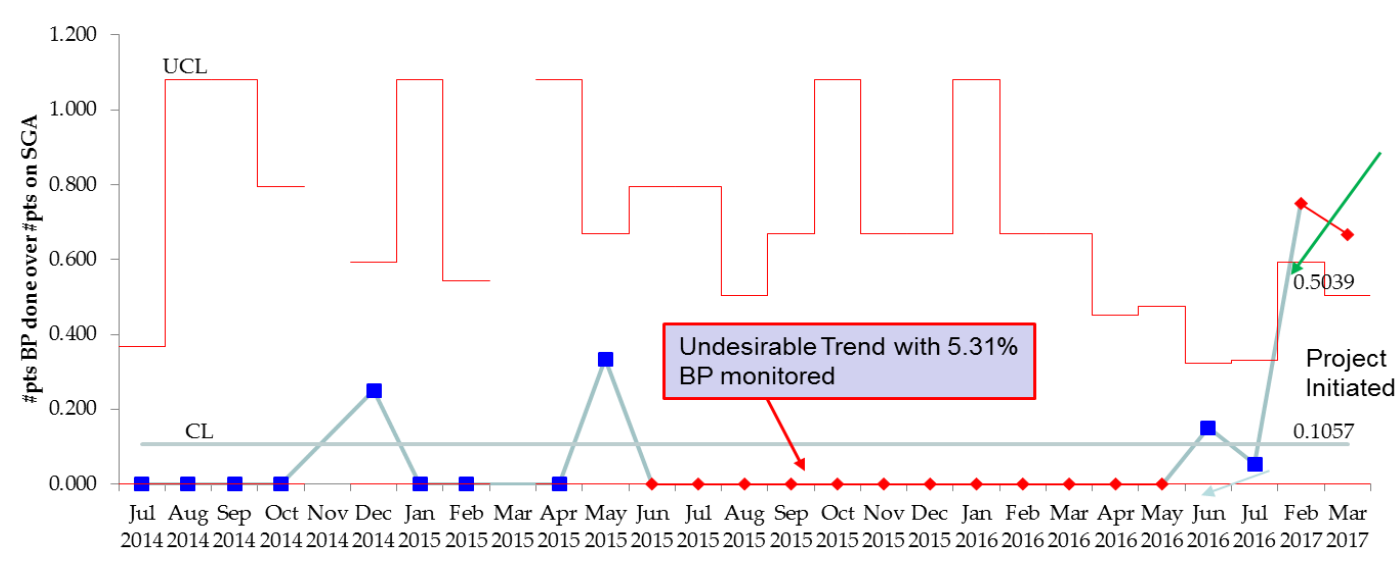


The control chart in Table 4 below compares pre-intervention data with the results of the post intervention data for BP and BMI. During the retrospective data collection time period it was business as usual as evidenced by the blue dots. Falling below the mean, an undesirable trend is noted by the red dots during the retrospective data collection period depicting a $0 \%$ baseline documentation rate of blood pressure and/or BMI. Following the educational intervention to the providers, a 58.23\% increase over baseline is noted well above the target outcome of a $20 \%$.increase.

Table 4 Rate of Patients Receiving Second Generation Antipsychotics
whose BP and BMI were Monitored per Standard of Care

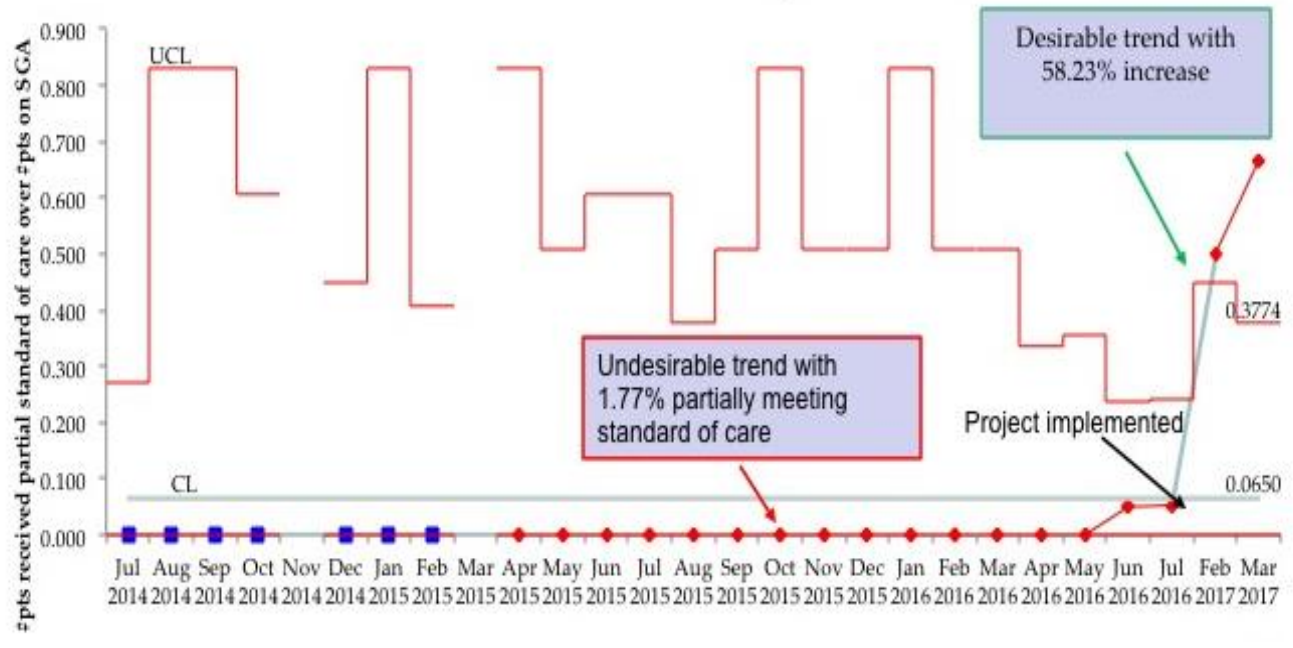

For the purposes of this pilot study, difficulty in ordering the disposable tape measures, provider reluctance, and provider time constraints influenced cost and value in continuing to pursue documentation of waist circumference in the electronic health record at this time. The stakeholders agreed that this data point was not feasible for the pilot study. Therefore, for this pilot study, the standard of care in regards to the ADA/APA/ACE/NAASO guideline was partially met. 


\section{Implications for practice}

The project heralded several unintended, yet significantly positive outcomes. Once it was established that blood pressure and BMI monitoring were to become a routine part of the psychiatric visit, a process protocol was developed in collaboration with the primary care and family medicine medical directors to identify and manage abnormal BP results. As the organization is moving towards Integrated Behavioral Healthcare delivery model with an emphasis on quality improvement, this level of collaboration between psychiatry and primary care was especially beneficial. Discussions were initiated on how the psychiatric clinic space could be more effectively used and a physical area was set aside for monitoring biometrics. The clinic director continues to the feasibility of utilizing a medical assistant from an alternate department to assist in the biometric collection on psychiatric clinic days. A representative from the Information Technology department noted that the consistent documentation of blood pressures, height and weight at the time of the psychiatric visit enhanced the organizations' participation in the Centers for Medicare and Medicaid Services Meaningful Use program. $^{12}$

Appreciating the concomitant implications for the providers in other areas of the clinic, this quality improvement project is the first step in the process of shifting psychiatric provider behavior to include adherence to the recommendations set forth in the guidelines for metabolic management of the patient prescribed SGA's. 


\section{Conclusions}

This evidence based practice project met its intended goal of increasing the psychiatric providers screening for metabolic changes by collecting baseline biometrics as recommended in the ADA/APA/ACE/NAASO consensus guideline. The adherence to the guideline is a sustainable addition to the routine care offered by the psychiatric clinic and a complement to the Integrated Behavioral Healthcare delivery model the organization has adopted. The successful implementation of the project may have long lasting impact on attention to metabolic changes within the behavioral health clinic patient population as well as integrate general healthcare screening into psychiatry practice for the psychiatric providers. 


\section{References}

1. American Diabetes Association, American Psychiatric Association, American Association of Clinical Endocrinologists, \& North American Association for the Study of Obesity. (2004). Consensus development conference on antipsychotic drugs and obesity and diabetes. Diabetes Care. 27(2), 596-601. https://doi.org/10.2337/diacare.27.2.596

2. Barnett, M., VonMuenster, S., Wehring, H., Popish, S., McDonald, K., Walker, V.M., Perry, P. (2010). Assessment of monitoring for glucose and lipid dysregulation in adult Medi-Cal patients newly started on antipsychotics. Annals of Clinical Psychiatry. 22(1): 9-18.

3. Dhamane, A.D., Matin, B.C., Brixner, D.I., Hudson, T.J., \& Said, Q. (2013). Metabolic monitoring of patients prescribed second generation antipsychotics. Journal of Psychiatric Practice, 19(5): 360-374. doi: 10.1097/01.pra.0000435035.45308.03

4. Dragatsi, D. \& Deakins, S. (2009). Implementing a metabolic initiative in a community mental health clinic. Psychiatric Services, 60(10): 1298-301. Doi: 10.1176/ps.2009.60.10.1298

5. Goff, D.C., \& Newcomer, J.W. (2007). Integrating general health care in private community psychiatry practice. Journal of Clinical Psychiatry, 68(7): 49-54.

6. Khan, A. Y., Shaikh, M.A., \& Ablah, E. (2010). To examine the extent of compliance to the proposed monitoring protocol among practicing psychiatrists for second generation antipsychotics. Journal of Pakistan Medical Association, 60(6): 446-50. 
7. Lloyd, S.T., D"Errico, E., \& Bristol, S.T. (2016). Use of the Iowa model of research in practice as curriculum framework for doctor of nursing practice (DNP) project completion. Nursing Education Perspectives 37 (1): 51-53. DOI: $10.5480 / 14-1364$

8. Lui, K., Randhawa, G., Totten, V., Smith, A. E., \& Raese, J. (2016). Is metabolic syndrome on the radar? Improving real-time detection of metabolic syndrome and physician response by computerized scan of the electronic medical record. The Primary Care Companion for CNS Disorders, 18(1), eCollection. doi: 10.4088/PCC.15m01849

9. McEvoy, J.P., Meyer, J.M., Goff, D.C., Nasrallah, H.A., Davis, S.M., Sullivan, L,...Lieberman, J.A. (2005). Prevalence of the metabolic syndrome in patients with schizophrenia: baseline results from the clinical antipsychotic trials of intervention effectiveness (CATIE) schizophrenia trial and comparison with national estimates from NHANES III. Schizophrenia Research, 80(1): 19-32. doi: $10.1016 / j . s c h r e s .2005 .07 .014$

10. Morrato, E.H., Druss, B., Hartung, D. M., Valuck, R. J., Allen, R., Campagna, E., Newcomer, J. W. (2010). Metabolic testing rates in 3 states Medicaid programs after FDA warnings and ADA/APA recommendations for second-generation antipsychotic drugs. Archives of General Psychiatry, 67(1): 17-24. doi: 10.1001/archgenpsychiatry.2009.179

11. Sernyak, M.J. (2007). Implementation of monitoring and management guidelines for second-generation antipsychotics. Journal of Clinical Psychiatry, 68 Suppl 4: $14-18$. 
12. Unutzer, J., Chan, Y.F., Hafer, E., Knaster, J., Shields, A., Powers, D., \& Veith, R.C. (2012). Quality improvement with pay-for-performance incentives in integrated behavioral healthcare. American Journal of Public Health. 102(6): e415. doi: 10.2105/AJPH.2011.300555 